\title{
Performance of Robotic Augmentation in Microsurgery-Scale Motions
}

\author{
Rajesh Kumar ${ }^{1}$, Tushar M. Goradia ${ }^{3}$, Aaron C. Barnes ${ }^{2}$, Patrick Jensen ${ }^{5}$, \\ Louis L. Whitcomb ${ }^{2}$, Dan Stoianovici ${ }^{2,4}$, Ludwig M. Auer ${ }^{6}$, and \\ Russell H. Taylor ${ }^{1}$ \\ ${ }^{1}$ Department of Computer Science \\ ${ }^{2}$ Department of Mechanical Engineering \\ ${ }^{3}$ Department of Neurosurgery \\ ${ }^{4}$ Department of Urology \\ ${ }^{5}$ Microsurgery Advance Design Laboratory \\ Johns Hopkins University, Baltimore, Maryland, USA, \\ ${ }^{6}$ Institute of Applied Sciences in Medicine (ISM-Austria)
}

\begin{abstract}
This paper is part of the development process of a microsurgical "cooperating" assistant. To evaluate its applicability to augment fine surgical motions, we test precision and operator perception in simple microsurgical scale pick and place motions. Such motions are common in microsurgical procedures(e.g. micro-vascular anastomosis). The experiments test the users' ability to position a common surgical tool to 250,200 and 150 micrometer accuracy. These experiments were performed using two test platforms. The new "steady hand" robot designed for microsurgery and the LARS robot (a laparoscopic camera holding robot) adapted for this purpose. Comparative results for several parameters including time, success rate, error rate, number of attempts are included. Comparison of performance of the two robots for these tasks is also included. The results support our claim that the new "steady hand" robot augments human performance for microsurgery-scale motion
\end{abstract}

\section{Introduction}

For the purpose of microsurgical applications, cooperative control systems promise significant advantages. A "steady hand" robot (e.g. [1]) can provide guidance, and enforce safety constraints. It can also use compliance models taking into account procedure-specific issues such as tissue properties and thereby provide tactile sensing for the human surgeon. A cooperative control system has the advantage of integrating safety and information integration into the procedure without taking away control from the human surgeon. This allows the surgeon's superior intelligence and experience to be used with greater precision and safety than before. 
A first step towards cooperating could be tremor reduction with a stiff robot. If such a system only is used to position surgical tools at the surgical site, the performance of the system would depend upon the performance of the robot and ease of use We discuss use of tremor reduction to increase accuracy of pick-and-place motions at the limits of human motor motion. These motions are required e.g. for micro-vascular surgery when small blood vessels are anastomosed. The task of anastomosis often involves the use of a very fine suture which is attached to a correspondingly small needle. The needle guides the suture material in and out of the vessel wall as the ends of the blood vessels are physically conjoined. Binocular magnification provides clear detail of the vessels and sutures but has little effect on the natural tremor of the surgeon. When the size of these tasks approaches the limit of human dexterity(10s of micrometers), tremor-reducing devices may provide benefit.

\subsection{Previous Work}

Lengthy procedures that require fine resolution motions are good candidates for robotic assistance $([2,3,4])$. Other minimally invasive tasks $([5,6])$ limit visual and proprioceptive feedback. Automation has already been applied in several procedures $([7,8])$ in neurosurgery. Other microsurgical fields have also seen increasing use of computer and imaging technology. Troccaz ([9]) designed a system that used a passive robotic arm to provide guidance with dynamic constraints. ACROBOT (Davies, [10]), Cobots (Peshkin, [11]) are other examples of synergistic systems. Active tremor cancellation ([12]), an alternative to passive compensation is also being investigated. Analysis and representation of tactile properties of tissues ([13]) and development of better tactile guidance systems are also current topics of research ([14]). Use of force and tactile feedback for guidance in simulators ([15]) and real robotic systems $([16,17,18])$ has also been published previously. Master-slave systems have been previously designed for use in microsurgical procedures $([19,20])$ and evaluated $([21,22])$. Human performance issues in these surgical procedures have also been studied $([23,24])$. Most performance evaluation studies of microsurgical tasks use time of completion as a major indicator ([25,26,27]). Simulators have also been used to compare/assess surgical skill ([28]). Since complex microsurgical tasks are difficult to quantify automated performance comparisons in the past have used visual inspection by the experimenter (e.g. [22]) or an observer. Authors have also previously looked at comparative performance issues ([29]). 


\section{Methods and Materials}

\subsection{Hardware}

We use the new "steady hand" robot ([1]) as the first platform for these experiments. Designed for microsurgical applications this is a 7-degree-of-freedom manipulator. It has $3 \mathrm{XYZ}$ translation stages at the base for coarse positioning, two rotational degrees of freedom at the shoulder (the RCM linkage, [30,31]), an instrument insertion and an instrument rotation stage. The instrument rotation stage was not used for this experiment. A force sensor (ATI Industrial Automation Inc.) is built in the end-effector. This force-sensor can be used to make the robot move in compliance to forces applied at the end-effector. This robot has a remote center of motion. It has an overall positional accuracy of 10 s of microns. It is a stiff and slow robot.

The second platform for these experiments was a LARS robot([32]). Designed for camera holding applications in laparoscopic surgery, LARS is a 7 degree-of-freedom manipulator. It has three translation stages at the base, two rotational degrees of freedom at the shoulder, an instrument insertion stage and an instrument rotation stage. LARS also has a force sensor built in the end-effector. LARS also has a remote center of motion. The robot has a stage resolution of 50 microns, and overall positional accuracy close to 100 microns. LARS is a slow robot and its upper linkages have only limited stiffness.

\subsection{Software}

The new "steady hand" robot uses a PC based controller system consisting of a DSP card for servo control and WIN32 operating system for application development. The modular robot control(MRC) library developed at JHU provides Cartesian level control. The application for these experiments was written using the library. LARS is controlled by a dedicated PC controller, and a more powerful remote PC computer networked to this controller provides the user and application interface.

We compare the accuracy and reliability of performing a highly precise task (repeated placement of microsurgical needle into various sizes of holes $(250 \mathrm{um}, 200 \mathrm{um}, 150 \mathrm{um})$ under magnified vision at 40-fold magnification). The experiment is performed by six users with and without the aid the robot as well as by the robots alone. The goal is to analyze long-term performance under the human stress-situation of working at the border of motor-space-resolution against time, while avoiding mistakes. Parameters are time to completion and number of errors per unit of time, or number of completed tasks in a given time vs. number of mistakes. 
The experiment was performed using common microsurgical tools and a datum surface. The surgical tool was a commonly used 10-0 microsurgical needle held with the help of a needle holder. The datum surface consists of a sandwich of two metallic sheets separated by an insulating surface. This sandwich contains patterns of holes of the same size placed 8 and $2 \mathrm{~mm}$ apart in interleaved fashion. These were duplicated for the three different sizes of the holes (250, 200 and 150 microns). The lower sheet is the success surface, and the upper the error surface. Both surfaces are connected to $\mathrm{I} / \mathrm{O}$ lines of the robot, and so is the microsurgical needle. Any closure of circuit generates a success or error event. An accounting algorithm keeps track of successes and errors. The thickness of the upper (error plate) is 100 microns, and the insulating surface is 50 microns thick. This makes the holes 150 microns deep. Up to 40 fold magnification microscope was used as a visual aid for the experiment. Three different versions of the pick-and-place experiments were performed. These versions were

1. Unassisted Series - the surgeon used only the conventional techniques to perform the pick-and-place operations

2. Hand Held Series - the needle holder was attached rigidly to the force sensor at the end-effector of the robot. The robot complied to user applied forces

3. Autonomous Series - the robot is registered to the plates and performs the task autonomously.

Autonomously, the robot was registered to eight corner holes of the pattern and interpolated the location of the rest. The users performed each of the first two modes of the experiment for a fixed interval of time. The robot was programmed to attempt pickand-place operations for the same interval of time. Data collection was automatic as the accounting algorithm monitored the digital inputs of robot to which the error and data surfaces were connected.

\section{Results}

Six different users performed the experiments for each robot. The hand-held manipulator version of the experiment resulted in better average success percentage than the conventional procedure performed by unaided user for the LARS robot. The LARS robot performed consistently with the same accuracy and better than all the three other modes for 200 and 150 microns autonomously. For the new "steady hand" robot, the robot outperformed all other modes and made very few mistakes. Users found both robots easy and convenient to use.

\begin{tabular}{|r|r|r|r|r|r|}
\hline \multirow{2}{*}{ Size of Holes } & \multirow{2}{*}{ Unassisted } & \multicolumn{2}{|c|}{ LARS Robot } & \multicolumn{2}{|c|}{ Steady Hand Robot } \\
\cline { 3 - 6 } & & Hand-held & Autonomous & Hand-held & Autonomous \\
\hline 250um & $48.8 \%$ & $56.0 \%$ & $53.0 \%$ & $77.8 \%$ & $98.4 \%$ \\
200um & $46.3 \%$ & $50.8 \%$ & $50.0 \%$ & $76.7 \%$ & $97.7 \%$ \\
$150 \mathrm{um}$ & $43.0 \%$ & $46.0 \%$ & $48.0 \%$ & $79.0 \%$ & $96.5 \%$ \\
\hline
\end{tabular}

Table 1. Comparison of performance in the three modes. The success rate (in \%) for microsurgical pick and place operations is given above. The new steady hand robot significantly improves the success rate for both assisted and autonomous modes. 
Table 1 compares the success rates of the three modes of the experiment. The average success percentages were $48.8,46.3$, and 43 percent for the three hole sizes in the unassisted series (single factor ANOVA, p<0.0001). The hand-held-manipulator series for LARS resulted in 56, 50.8, and 46 percent successes (Single factor ANOVA, $\mathrm{p}<0.02$ ) and reduced the errors significantly (paired t-test, $\mathrm{p}<0.01$ ). Size of holes did not significantly influence the result. The new "steady hand" robot, the hand-held-manipulator series resulted in 77.8, 77.6, and 79.0 percent successes and size of holes did not significantly influence the performance.

\begin{tabular}{|r|r|r|r|r|r|}
\hline \multirow{2}{*}{ Size of Holes } & \multirow{2}{*}{ Unassisted } & \multicolumn{2}{|c|}{ LARS Robot } & \multicolumn{2}{c|}{ Steady Hand Robot } \\
\cline { 3 - 6 } & & Hand-held & Autonomous & Hand-held & Autonomous \\
\hline 250um & 120.5 & 65.0 & 58.0 & 29.6 & 5.0 \\
200um & 110.0 & 76.8 & 62.0 & 27.0 & 7.0 \\
150um & 107.0 & 66.8 & 65.0 & 25.0 & 11.0 \\
\hline
\end{tabular}

Table 2. Average Number of Erroneous attempts for the experiment. The new steady hand robot significantly decreases errors for all pick and place motions.

Table 2 lists the number of erroneous attempts. For LARS robot, the total number of attempts as well as the average success percentages decreased with decreasing hole size, except autonomously where the LARS robot performed the same number of attempts with decreasing number of successes. This could be attributed to increase in the difficulty of the task with decreasing hole diameter.

For the new "steady hand" robot, the number of attempts, and success percentage remained approximately constant. This is because of superior accuracy of the robot, so the decreasing size of holes did not pose a significant problem. The new "steady hand" robot-human system therefore has a better accuracy than measured using untrained, unskilled users in this experiment. Further tests will need to be conducted with smaller motions and skilled, trained users to better estimate the accuracy of the system.

\begin{tabular}{|r|r|r|r|r|r|}
\hline \multirow{2}{*}{ Size of Holes } & \multirow{2}{*}{ Unassisted } & \multicolumn{2}{|c|}{ LARS Robot } & \multicolumn{2}{c|}{ Steady Hand Robot } \\
\cline { 3 - 6 } & & Hand-held & Autonomous & Hand-held & \multicolumn{1}{c|}{ Autonomous } \\
\hline 250um & 236.5 & 148.5 & 125.0 & 129.0 & 315.0 \\
200um & 206.1 & 149.6 & 125.0 & 107.0 & 311.0 \\
150um & 189.3 & 144.1 & 125.0 & 107.3 & 320.0 \\
\hline
\end{tabular}

Table 3. Total Number of attempts for the experiment. These are average number of trials completed over 10 minutes.

Table 3 compares the total number of attempts for various modes of the experiment and table 4 shows average times between attempts. The larger time required for the new "steady hand" robot is expected in part due to the simple force proportional velocity control used. However, given the superior success rates, in this case slow motion is justified. 


\begin{tabular}{|r|r|r|r|r|r|}
\hline \multirow{2}{*}{ Size of Holes } & \multirow{2}{*}{ Unassisted } & \multicolumn{2}{|c|}{ LARS Robot } & \multicolumn{2}{c|}{ Steady Hand Robot } \\
\cline { 3 - 6 } & & Hand-held & Autonomous & Hand-held & Autonomous \\
\hline 2.7 & 4.1 & 4.8 & 4.6 & 1.9 \\
200um & 2.9 & 4.0 & 4.8 & 5.6 & 1.9 \\
150um & 3.3 & 4.2 & 4.8 & 5.6 & 1.9 \\
\hline
\end{tabular}

Table 4. Average time between attempts (in seconds). The increase in time with new steady hand robot as a hand held manipulator may be due to the lack of training and the simple force proportional velocity control used.

For autonomous series, LARS could not be programmed to go any faster and still achieve good results because of its limited accuracy. The new "steady hand" robot is faster than all other modes of the experiment. The mistakes made by the robot autonomously are due to the simple control (PD control for positioning), and manufacturing tolerances in the datum grid.

\section{Discussion and Conclusions}

Present experience shows that the robotic system is less prone to mistaken movement patterns and can perform the given task repeatedly with the same performance. The results thus suggest that the robot can extend human capabilities by assisting with microsurgical tasks at a greater spatial resolution than humanly possible. As a consequence, the robot may improve the safety of delicate microsurgical procedures at very high magnification, where human motor-skill is artificial and overall space-orientation is also lost. In addition, given the well-known phenomenon of human tiring and loss of attention with increasing time, robotic assistance may benefit lengthy microsurgical procedures.

LARS has a relatively high stage resolution of 50 microns and positional accuracy close to 100 microns: two-thirds the size of smallest holes. Also, it has limited stiffness in its arm linkages. The new "steady hand" robot significantly increases the pick and place accuracy without significantly affecting the operator perception(from reported ease of use). Training is also likely to be a factor (as previous studies have also reported, e.g. [22] ), the users were mostly computer science graduate students with no robotic or microsurgical training. Their unfamiliarity with motions at this scale suggests that performance would improve with skilled and trained users.

Several other factors such as mistakes made in positioning the needle, spacing between sutures, etc are important quantifiers of surgical skill. These factors are difficult to evaluate without an automated testing platform. Further experiments in evaluating these and other parameters are planned. 


\section{Summary}

Several groups, including Davies ([10]), Troccaz ([9]), Peshkin ([11]), and ours have been exploring cooperative manipulation approaches to surgery. There have also been a number of telesurgical systems proposed for microsurgery (e.g. $[19,20,21])$. This paper reports the first experiments we are aware of to quantitatively assess the performance enhancement of microsurgical manipulation using the cooperative approach.

\section{Acknowledgements}

The authors gratefully acknowledge the support of the National Science Foundation under grant \#IIS9801684, the Engineering Research Center grant \#EEC9731478. Some of this work was also supported in part by NSF Grant \#5T32HL07712 from the NSF Whitaker Foundation Program for Cost Reduction in Health Care, and by The Johns Hopkins University internal funds. The LARS robots were produced by IBM research and donated to The Johns Hopkins University as part of a generous equipment grant. We also acknowledge the support of the users for these experiments.

\section{References}

1 Taylor R.H, Barnes A, Kumar R, Gupta P, Jensen P, Whitcomb L.L, de Juan E., Stoianovici D., Kavoussi L, A Steady-Hand Robotic System for Microsurgical Augmentation, accepted for publication in MICCAI'99.

2 Charles S T, Schenker P S et al., Development of a Telemanipulator for dexterity enhanced Microsurgery, MRCAS'95, Baltimore, USA, pp81-88

3 Charles Steve, Dexterity Enhancements for Surgery in Computer Integrated Surgery, MIT press. pp467-471.

4 Kelly Patrick J, Computer-Assisted Neurosurgery in Computer Integrated Surgery, MIT press, pp301-306.

5 Auer L M, Deinsberger W, NiederKorn K, et al., Endoscopic versus medical treatment of spontaneous intracerebral hemorrhage, Journal of Neurosurgery, 1989, 70:pp 530-535.

6 Auer, L.M., Robots for Neurosurgery?, Minimally Invasive Techniques for Neurosurgery: Current Status and Future Perspectives,.eds. Bauer, B.L., Hellwig, D., Springer Verlag, 1998, pp. 243-249.

7 Glauser D, Fankhauser H, Epitaux M, Hefti J L, Jaccotte A, neurosurgical robot minerva - final results and current developments, MRCAS'95, Baltimore, USA, pp24-30.

8 Ryan M J, Erickson R K, Levin D N, Pelizzari C A, MacDonald R L, Dohrmann G J, Frameless stereotaxy with real-time tracking of patient head movement and retrospective patient image registration, MRCAS'95, Baltimore, USA, pp1-7.

9 Delnondedieu Y, Troccaz J, PADyC: a passive arm with dynamic constraints. A prototype with two degrees of freedom, MRCAS'95, Baltimore, USA, pp173-180.

10 Davies B.L., Fan K.L., Hibberd R.D., Jakopec M., Harris S.J., ACROBOT - Using Robots and Surgeons Synergistically in Knee Surgery, 8th International Conference on Advanced Robotics, CA, USA, 1997 
11 Colgate J.E., Wannasuphoprasit W., Peshkin M.A., Cobots: Robots for Collaboration with Human Operators, Proceedings of the International Mechanical Engineering Congress and Exhibition, Atlanta, GA, 1996, DSC-Vol. 58, pp. 433-39.

12 Riviere C N, Rader R S, Thakor N V, Adaptive real-time canceling of physiological tremor for microsurgery, MRCAS'95, Baltimore, USA,pp89-96.

13 Basdogan C, Ho C-H, Srinivasan M A, Small S D, Dawson S L, Force Interactions in Laparoscopic Simulations: Haptic Rendering of Soft Tissues, MMVR'98, San Diego, US, pp.385-391.

14 Kontarinis D A, Howe R D, Tactile display of vibratory information in teleoperation and virtual environments, Presence, 1995, 4(4):pp. 387-402.

15 Yagel R, Stredney D, et al, Multisensory Platform for Surgical Simulation, IEEE virtual Reality Annual International Symposium, Santa Clara, US, 1996, pp. 72-78.

16 Taylor R H, Funda J, LaRose D, Treat M, A telerobotic system for augmentation of endoscopic surgery, IEEE Engineering in medicine and biology society, 1992, pp. 1054-1056.

17 Lavallee J, Troccaz S, Gaborit L, Cinquin P, Benabid A L, Hoffman D, Image guided robot: a clinical application in stereotactic neurosurgery. IEEE Int. conf. on Robotics and Automation, Nice, France, 1992, pp. 618-625.

18 Troccaz J, Peshkin M, Davies B L, The use of localizers, robots and synergistic devices in CAS, CVRMed-MRCAS'97, Grenoble, France, pp. 727-736, 1997.

19 Mitsuishi M, Watanbe H, Nakanishi H, Kubota H, Izuka Y, Dexterity enhancement for a tele-micro-surgery System with Multiple Macro-micro Co-located Operation Point Manipulators and Understanding of the Operator's Intention, CVRMed-MRCAS'97, Grenoble, France, pp. 821-830, 1997.

20 Mitsuishi M., Iizuka Y. Remote Operation of a Micro-Surgical System, Proceedings of IEEE International Conference on Robotics and Automation(ICRA'98), Leuven, Belgium 1998

21 Krapohl B. D., Zins J .E., Siemionow M., Computer Assisted Microsurgery: Introduction of a new robotic arm, $4^{\text {th }}$ International Symposium on Computer Assisted Orthopedic Surgery, Davos, Switzerland,March 1999.

22 Salcudean S.E., Ku S., Bell G., Performance Measurement in Scaled Teleoperation for Microsurgery, Proceedings of CVRMed-MRCAS'97, Springer-Verlag Lecture Notes in Computer Science, March 1997, Vol. 1205, pp.789-798.

23 Sheridan Thomas B, Human factors in Tele-inspection and Tele-surgery:Cooperative manipulation under Asynchronous Video and Control Feedback, Proceedings of MICCAI'98, Springer-Verlag Lecture Notes in Computer Science, October 1998, Vol. 1496, pp368-376.

24 Vasilakos K., Glass L., Beuter A., Interaction of tremor and magnification in motor performance task with visual feedback, Journal of Motor Behavior, June 1998, Vol.30(2),pp 158-168.

25 Starkes, Janet L., Payk Irene, Hodges Nicola J., Developing a standardized test for the assessment of suturing skill in novice microsurgeons, Microsurgery, 1998, Vol 18, pp.1922.

26 Tendick F., Bhoyrul S., Way L. Comparison of Laparoscopic Imaging Systems and Conditions Using a Knot Tying Task, Journal of Image Guided Surgery, vol. 2, no. 1, 1996.

27 Kavoussi L.R, Moore R. G., Adams J. B., Partin A. W., Comparison of robotic versus human laparoscopic camera control, Proceedings of MRCAS'95,Baltimore, USA, 1995, pp284-287. 
28 O'Toole R., et al, Assessing Skill and Learning in Surgeons and Medical Students Using a Force Feedback Surgical Simulator, Proceedings of MICCAI'98, Springer-Verlag Lecture Notes in Computer Science, October 1998, Vol. 1496, pp.899-909.

29 Kumar R, Goradia T M, Taylor R H, Auer L M, Robot-Assisted Minimally-Invasive Neurosurgical Procedures: Dexterity Experiments, SIMT'97, Kyoto, Japan.

30 Stoianovici D., et al, An Efficient Needle Injection Technique and Radiological Guidance Method for Percutaneous Procedures, CVRMed-MRCAS'97, Grenoble, France March 1997.

31 Stoianovici, D., Whitcomb, L.L., Anderson J.H., Taylor R.H., Kavoussi L.R., A Modular Surgical Robotic System for Image Guided Percutaneous Procedures, Proceedings of MICCAI'98, Lecture Notes in Computer Science, Springer-Verlag, ,1998 Vol. 1496, pp.404-410.

32 Taylor, R.H., et al., A Telerobotic Assistant for Laparoscopic Surgery, in ComputerIntegrated Surgery, eds R. Taylor, et al., , MIT Press. 1996, pp581-592. 